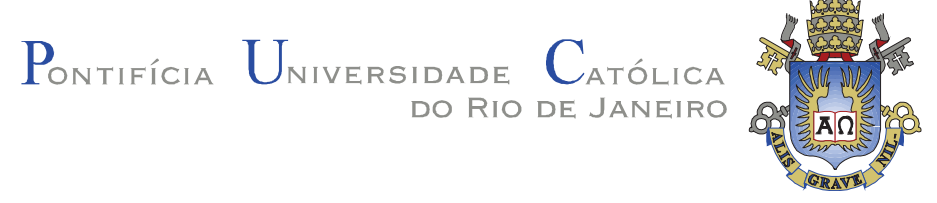

Letícia do Carmo Titoneli

\begin{abstract}
Modelagem e Simulação de Eventos Discretos do Sistema Logístico de um Terminal Portuário com Identificação de Gargalos Gerados por Ineficiência dos Processos Operacionais.
\end{abstract}

Dissertação apresentada como requisito parcial para obtenção do grau de Mestre pelo Programa de PósGraduação em Engenharia de Produção do Departamento Engenharia Industrial da PUC-Rio.

Orientador: Prof. Fabrício Oliveira 


\section{Modelagem e Simulação de Eventos Discretos do Sistema Logístico de um Terminal Portuário com Identificação de Gargalos Gerados por Ineficiência dos Processos Operacionais.}

Dissertação apresentada como requisito parcial para obtenção do grau de Mestre pelo Programa de PósGraduação em Engenharia de Produção do Departamento Engenharia Industrial da PUC-Rio. Aprovada pela Comissão Examinadora abaixo assinada

Prof. Fabrício Oliveira Orientador e Presidente Departamento de Engenharia Industrial - PUC-Rio

Prof. José Eugênio Leal Departamento de Engenharia Industrial - PUC-Rio

Prof. Fernando Luiz Cyrino Oliveira Departamento de Engenharia Industrial - PUC-Rio

Prof. José Eugênio Leal Coordenador Setorial do Centro Técnico Científico - PUC-Rio 
Todos os direitos reservados. É proibida a reprodução total ou parcial do trabalho sem a autorização da universidade, do autor e do orientador.

\section{Letícia do Carmo Titoneli}

Graduou-se em Engenharia de Produção pela Universidade Federal Fluminense - UFF em 2010. Atuou nas áreas de logística, indústria automobilistística e planejamento estratégico. Atualmente, é Especialista em Operações Portuárias no Terminal de Contêineres - Sepetiba Tecon, empresa coligada ao grupo CSN, onde desenvolve estudos de capacidade, dimensionamento de mão de obra, equipamentos e investimentos.

Ficha Catalográfica

Titoneli, Letícia do Carmo

Modelagem e simulação de eventos discretos do sistema logístico de um terminal portuário com identificação de gargalos gerados por ineficiência dos processos operacionais / Letícia do Carmo Titoneli; orientador: Fabrício Oliveira. - 2015.

80 f.: il. (color.); $30 \mathrm{~cm}$

Dissertação (mestrado)-Pontifícia Universidade Católica do Rio de Janeiro, Departamento de Engenharia Industrial, 2015.

Inclui bibliografia

1. Engenharia Industrial - Teses. 2. Simulação. 3. Gargalos do sistema lógico. 4. Terminal de contêiner. I. Oliveira, Fabrício. II. Pontifícia Universidade Católica do Rio de Janeiro. Departamento de Engenharia Industrial. III. Título.

CDD: 658.5 
Dedico este trabalho primeiramente a DEUS e aos meus pais, Sueli e Antônio, que tanto amo e que me deram forças em vários momentos. Dedico também à minha irmã Daniele, pelo ombro amigo nas horas difíceis. 


\section{Agradecimentos}

Ao professor Fabrício Oliveira, pela dedicação à minha orientação, pela paciência e incentivo durante a elaboração deste trabalho.

Ao Gerente Geral de Operações de Contêineres, Cesar Maas, pelos ensinamentos e apoio nos momentos necessários.

À Sepetiba Tecon, por ter possibilitado o acesso aos dados para o desenvolvimento do modelo de simulação.

Ao consultor Isac Santana, da Paragon, que auxiliou-me nas dúvidas de estruturação do modelo de simulação.

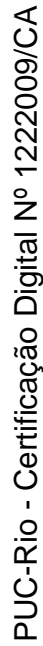

E a todos os amigos que me apoiaram no início dessa caminhada. 


\section{Resumo}

Titoneli, Letícia do Carmo; Oliveira, Fabrício. Modelagem e simulação de eventos discretos do sistema logístico de um terminal portuário com identificação de gargalos gerados por ineficiência dos processos operacionais. Rio de Janeiro, 2015. 80p. Dissertação de Mestrado Departamento de Engenharia Industrial, Pontifícia Universidade Católica do Rio de Janeiro.

O crescimento do mercado de contêineres e a busca dos terminais pela melhoria em seus processos portuários tem obtido a atenção da área científica. Com enfoque nos estudos de simulação, o presente trabalho tem por objetivo modelar e simular um sistema logístico de um terminal portuário, cuja especialização é a movimentação de contêineres. Foram desenvolvidos 6 cenários com finalidade de avaliar os recursos necessários capazes de movimentar um volume específico de contêineres por uma unidade de tempo. O modelo é construido no software ARENA e a análise dos resultados realizada através dos indicadores comuns em terminais de contêineres. Esses indicadores são analisados para cada um dos cenários desenvolvidos e como conclusão avalia-se a capacidade instalada para cada um dos cenários. Para validação do modelo foram construídos 2 cenários e os outros 4 cenários avaliaram como os recursos de três berços de atracação de navios, 6 Portêineres, 6 Rubber Tyred Gantry, 11 Reach Stackers, 26 carretas, 4 Gates , 1 Scanner e 30 slots de Inspeção de comportam com a movimentação de 440.000 contêineres/ano e 660.000 contêineres/ano.

\section{Palavras chave}

Simulação; Gargalos do Sistema Logístico; Terminal de Contêiner. 


\section{Abstract}

Titoneli, Letícia do Carmo; Oliveira, Fabrício (Advisor). Modeling and simulation of discrete in a event logistics system of a port terminal with identifying bottleneck generated by inneficient operational process. Rio de Janeiro, 2015. 80p. MSc. Dissertation - Departamento de Engenharia Industrial, Pontifícia Universidade Católica do Rio de Janeiro.

The growth of the container's market and the search of terminals for the improvement in their port processes has gotten the attention of the scientific area. With focus on simulation studies, the present work aims to model and simulate a logistic system of a port terminal, whose specialization is the containers movements. Six scenarios have been developed in order to assess the resources needed to handle a specific volume of containers by a unit of time. The model is built in the software ARENA and the analysis of results was performed through common indicators in container terminals. These indicators were analyzed for each of the scenarios developed and finally evaluated the installed capacity for each of the scenarios. For de model validation were built two scenarios and for the other four sceanrios assessed how the resources of three berths for ships, 6 ship to shore, 6 rubber tyred gantry, 11 reach stackers, 26 trucks, 4 gates, 1 scanner and 30 inspection slots behaves with 440.000 container/year and 660.000 container/year.

\section{Keywords}

Simulation; Logistics System Bottlenecks; Container Terminal. 


\section{Sumário}

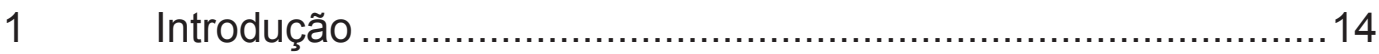

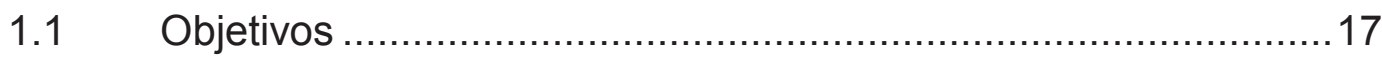

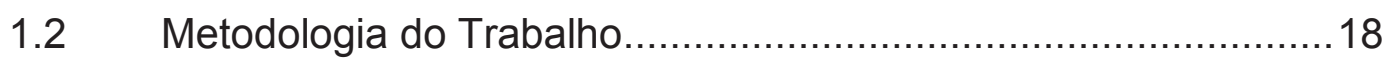

1.3 Delimitações do Tema..........................................................19

$1.4 \quad$ Estrutura Apresentada .......................................................

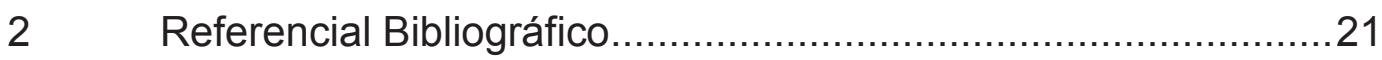

2.1 Método Matemático - Otimização..............................................21

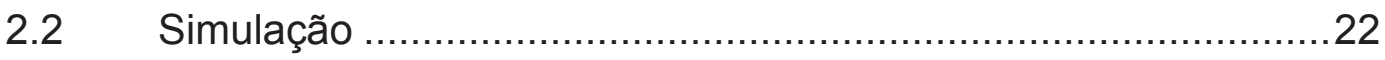

2.2.1 Simulação de Cenários Específicos - Objetivo único.............23

2.2.2 Simulação - Objetivo Macro ...............................................26

2.3 Conclusões sobre o Referencial Bibliográfico ............................27

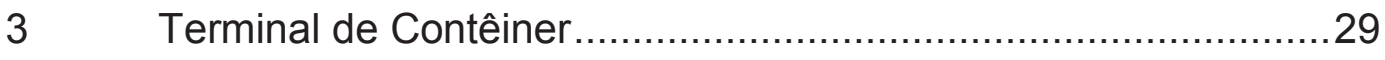

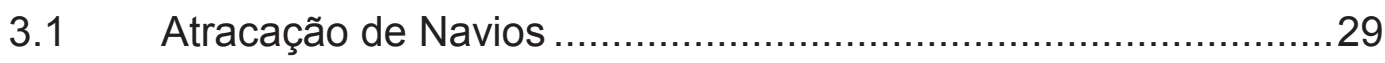

3.2 Descarregamento e Carregamento de Navios ............................32

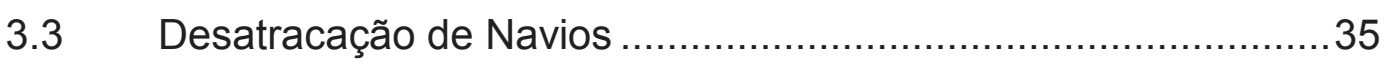

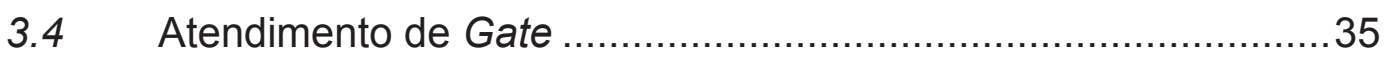

3.5 Atendimento dos Órgãos Anuentes ......................................... 36

4 Mapeamento dos Processos de Terminal de Contêiner...............39

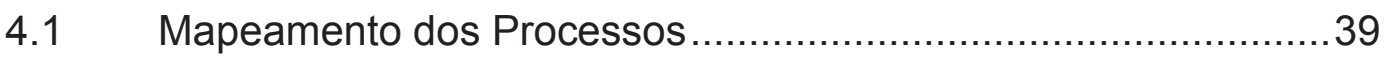

4.1.1 Atendimento de Gate ...................................................... 39

4.1.2 Carregamento/Descarregamento de Navio .........................41

4.1.3 Escaneamento de Contêineres...........................................42

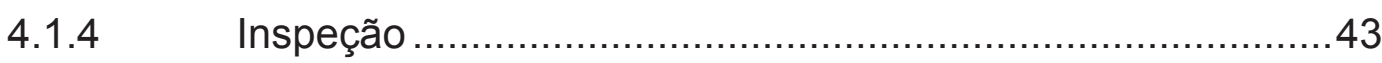

5 Simulação de um Terminal de Contêiner ....................................44 
5.1 O Terminal de Contêiner Sepetiba Tecon -

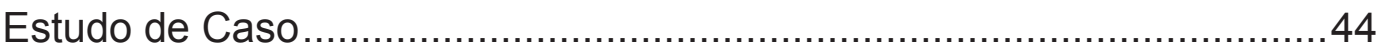

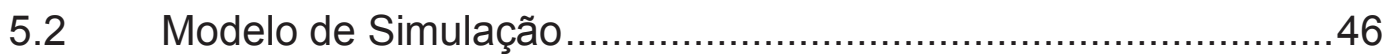

5.2.1 Etapa 1: Concepção ou Formulação do Modelo....................46

5.2.1.1 Premissas do Modelo ..................................................... 47

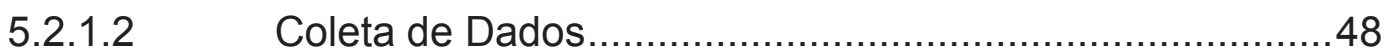

5.2.2 Etapa 2: Implementação do Modelo ....................................49

5.2.2.1 Verificação e Validação do Modelo ....................................50

5.2.3 Etapa 3: Análise dos Resultados do Modelo .......................52

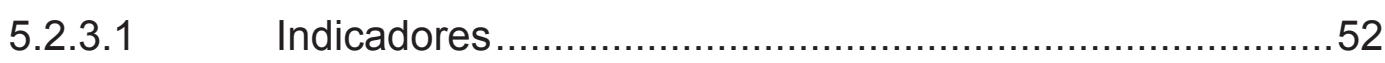

5.2.3.2 Resultados dos Cenários .............................................56

5.2.3.3 Análise dos Resultados ..................................................57

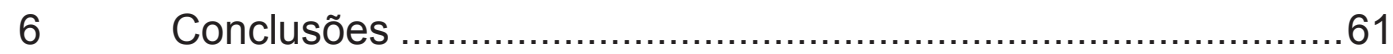

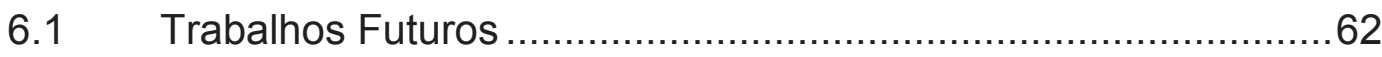

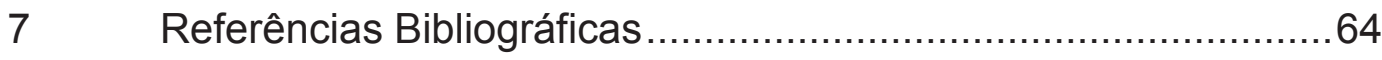

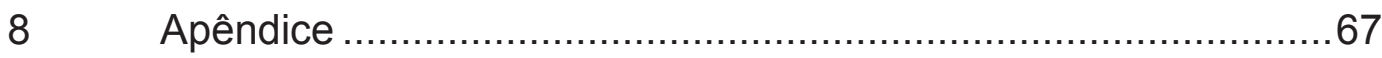

8.1 Apêndice 1 - Algumas Distribuições dos

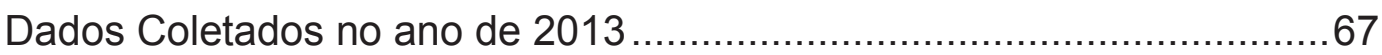

8.2 Apêndice 2 - Lógica dos Processos Construídos .......................73 


\section{Lista de Figuras}

FIgURA 1 - CLASSIFICAÇÃO dOS TIIOS DE PESQUISAS BIBLIOGRÁFICAS..........18

Figura 2 - Mapa Janela Terminal ContÊInERES SePetiba TECon.............30

Figura 3 - Line Up do terminal SepetiBA TeCon 02/06 A 08/06 ................31

FIGURA 4 - QUADRO DE EQUIPAMENTOS ............................................ 33

FIGURA 5 - VARA DE PEAÇÃO ........................................................... 34

FIGURA 6 - VEHICLE MOUNT TERMINAL .............................................. 34

FIGURA 7 - LAYOUT DO TERMINAL CONTÊINER -

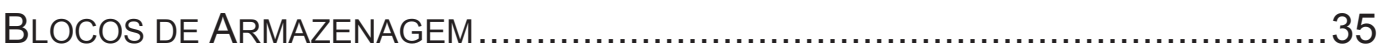

FiguRA 8 - JANELA" DE AgENDAMENTO NO WEBSite NO

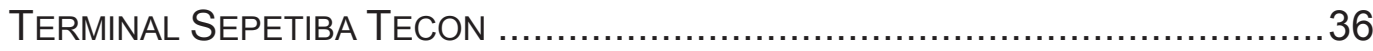

FIgURA 9 - GALPÃO dE INSPEÇÃO dE MERCADORIAS/CARGAS E

SCANNER

FIgURA 10 - ETAPAS dO ATENDIMENTO DE GATE DE EXPORTAÇÃO ................40

FIgURA 11 - ETAPAS DO ATENDIMENTO DE GATE DE IMPORTAÇÃO.................41

FIGURA 12 - ETAPAS DO CARREGAMENTO/DESCARREGAMENTO

DE NAVIO -

FIgURA 13 - ETAPAS DE ESCANEAMENTO DE CONTÊINER ...........................42

FIGURA 14 - ETAPAS DA INSPEÇÃO DE CONTÊINER .................................. 43

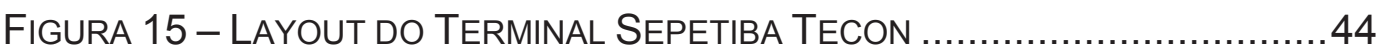

FiguRA 16 - Fotografia AÉREA do SEpetiba TECon $\ldots \ldots \ldots \ldots \ldots \ldots \ldots \ldots \ldots . \ldots . \ldots \ldots$

Figura 17 - Metodologia de Simulação - Adapatado ChWif e MedinA, 2013

FIGURA 18 - ARENA - OPERAÇÃO DE EXPORTAÇÃO - PARTE $01 \ldots \ldots \ldots \ldots \ldots . . . . . .73$

FIGURA 19 - ARENA - OPERAÇÃO DE EXPORTAÇÃO - PARTE $02 \ldots \ldots \ldots \ldots \ldots . . . . . .73$

FIGURA 20 - ARENA - OPERAÇÃO DE EXPORTAÇÃO - PARTE 03 ...................73

FIGURA 21 - ARENA - OPERAÇÃO DE EXPORTAÇÃO - PARTE $04 \ldots \ldots \ldots \ldots \ldots . . . . . .73$ 
FIGURA 22 - ARENA - OPERAÇÃO DE EXPORTAÇÃO - PARTE $05 \ldots \ldots \ldots \ldots \ldots \ldots \ldots . . . .73$

FIGURA 23 - ARENA - OPERAÇÃO DE IMPORTAÇÃO - PARTE 01 .....................74

FIGURA 24 - ARENA - OPERAÇÃO DE IMPORTAÇÃO - PARTE $02 \ldots \ldots \ldots \ldots \ldots \ldots . . . . . .74$

FIGURA 25 - ARENA - OPERAÇÃO DE IMPORTAÇÃO - PARTE 03 ......................74

FIGURA 26 - ARENA - OPERAÇÃO DE IMPORTAÇÃO - PARTE 04 ......................74

FIGURA 27 - ARENA - OPERAÇÃO DE IMPORTAÇÃO - PARTE $05 \ldots \ldots \ldots \ldots \ldots \ldots \ldots . . . . .75$

FIGURA 28 - ARENA - OPERAÇÃO DE IMPORTAÇÃO - PARTE 06 …..................75

FIGURA 29 - ARENA - OPERAÇÃO DE IMPORTAÇÃO - PARTE $07 \ldots \ldots \ldots \ldots \ldots \ldots . . . . . . .75$

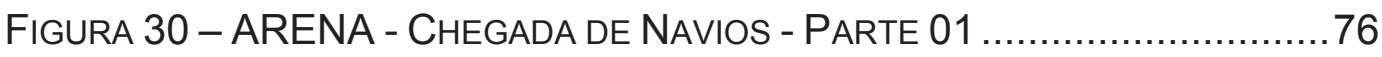

FIGURA 31 - ARENA - ChegadA DE NAVIOS - PARTE 02 …........................76

FIGURA 32 - ARENA - ChegadA DE NAVIOS - PARTE 03 ............................76

FIGURA 33 - ARENA - CHEGADA DE NAVIOS - PARTE $04 \ldots \ldots \ldots \ldots \ldots \ldots \ldots \ldots \ldots . . . . . . . . . . . . . . . .77$

FIGURA 34 - ARENA - ChegAdA DE NAVIOS - PARTE 05 ...........................77

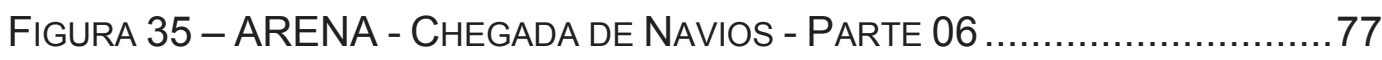

FIGURA 36 - ARENA - OPERAÇÃo TRANSBORdo - PARTE $01 \ldots \ldots \ldots \ldots \ldots \ldots . . . . . . . . . . . . .78$

FIGURA 37 - ARENA - OPERAÇÃo TRANSBORDo - PARTE 02 ………............78

FIGURA 38 - ARENA - OPERAÇÃo TRANSBORDO - PARTE 03 ……...............78

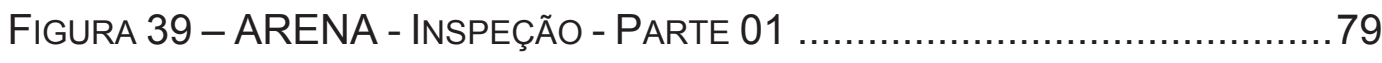

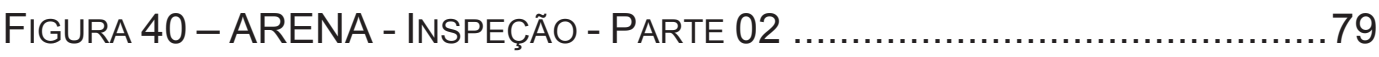

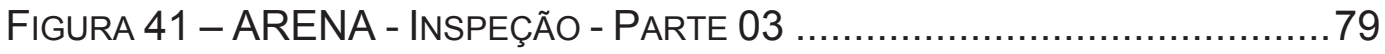

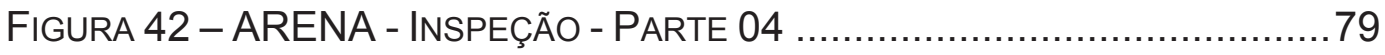

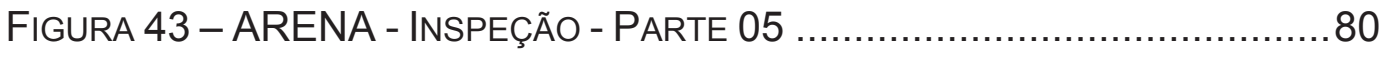

FIGURA 44 - ARENA - ENTRADA E SAÍDA DE DADOS - PARTE $01 \ldots \ldots \ldots \ldots \ldots \ldots . . . . . . . . . . .80$ 


\section{Lista de Tabelas}

TABELA 1 - QUAdRo SínTESE dAS REFERÊNCIAS BIBLIOGRÁFICAS ................27

TABELA 2 - DAdOS DE ENTRAdA DO MOdELO..........................................4 48

TABELA 3 - DistRIBUIÇÕES dos DAdos ColetAdos ....................................49

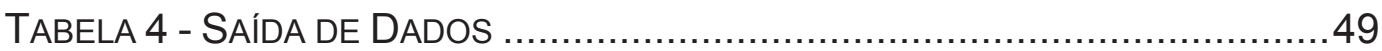

TABELA 5 - Modelo Construído EM ARENA .......................................50

TABELA 6 - DAdOS DAS REPLICAÇÕES DO ANO DE 2013 .............................51

TABELA 7 - RESULTADOS DOS CENÁRIOS ............................................... 56 
"Quem planeja a curto prazo deve cultivar cereais; a médio prazo deve plantar árvores; a longo prazo, educar homens."

Kwantsu Séc 3 aC 